\title{
Emergence of an Information Infrastructure through Integrating Waste Drug Recycling, Medication Management, and Household Drug Management in China
}

\author{
Yumei Luo \\ College of Business and Tourism Management, \\ Yunnan University \\ luoyumei@hotmail.com
}

\author{
Kai Reimers \\ School of Business and Economics \\ RWTH Aachen University \\ reimers@wi.rwth-aachen.de
}

\begin{abstract}
The total amount of waste drugs is expanding significantly as populations age and societies become wealthier. Drug waste is becoming a problem for health and environment. Thus, how to reduce and effectively dispose of waste drugs is increasingly becoming an issue for society. In this paper, we analyze the current situation with regard to existing systems for expired drug recycling and disposal in China and suggest that by connecting the involved practices of waste drug recycling, medication management, and household drug management the incentives to participate in an integrated drug recycling system can be dramatically increased for all involved actors. This is important from an IS perspective because such connecting of practices could account for a novel mechanism of information infrastructure emergence, known from physics as 'state transition'. By contrast, the current literature focuses on the development of information infrastructures through growth at the periphery, mostly driven by user activity and enabled by modular and flexible designs. Our approach could explain the de-novo emergence of an information infrastructure.
\end{abstract}

\section{Introduction}

Household waste medicines refers to medicine waste residing within homes [1]. Such waste does not only cause economic loss but has also an adverse impact on the environment and on human health if the waste is improperly disposed of [2]. Specifically, medical substances have been detected in sewage effluents [3, 4], surface water [5], groundwater, and even drinking water [6]. Safety is also compromised when medicines fall into the hands of children or of individuals who wish to abuse the medicines themselves or to sell expired medicines on a "grey" market.
In China, storing drugs for emergent use in homes in large quantities and varieties has become a common practice. With the rapid economic and social development of society the total amount of household waste drugs is expanding significantly. In 2014, a survey showed that about $78.6 \%$ of families in China have the habit of storing prescription medicine and more than $80 \%$ of the families do not regularly purge their stock from expired medicines and even fewer people know how to properly dispose of the expired medicine [7]. The survey also showed that $72.5 \%$ of families throw expired drugs into regular household waste, $5 \%$ flush them down the toilet or drain, and 6\% of families even continue to use them. Therefore, how to dispose of expired drugs has become an important issue for government and society. However, systems and processes for recycling expired drugs are still in an early stage in China. Although the government and some enterprises have implemented such processes and systems, most of these activities are carried out by public welfare organizations with government playing only a promoting and guiding role, which tends to be ineffective with regard to coordinating various stakeholders and to be limited in duration and range. In the current recycling regime, there are no obligations or incentives for enterprises to collect and for consumers to return expired drugs. Thus, it is urgent to ask how to effectively reduce and dispose of expired drugs through better coordination among the various actors involved in drug-related activities.

The accumulation of expired drugs in family homes is the result of a number of factors encompassing: (1) patients recovering before their dispensed medicines have all been taken; (2) therapies being stopped or changed because of ineffectiveness and/or unwanted side effects; (3) patients' deaths; (4) factors relating to repeat prescribing and dispensing processes [8].

Pharmaceuticals are special and therefore cannot be treated like other commodities [9]. Reasons for the special character of drugs include the high cost and 
long duration of research and development and the repercussions of the product not being available. This special character of drugs has implications for how to properly deal with expired drugs. On the one hand, expired drugs, when remaining unopened in their original container, would be expected to remain stable for an average of 57 months after their expiration [10]. An extreme case concerns Phenytoin (Dilantin), an anti-seizure medication, which remains effective for 20 years following its expiration. On the other hand, some drugs, such as antibiotics, blood thinners, and chemotherapy drugs can become toxic and hazardous to human health and the environment if they are not disposed of properly [11]. Thus, effective practices to reduce drug waste include not only improving the use of drugs, but also the effective disposal of expired/unwanted drugs. In sum, it is urgent to reduce preventable medicine waste and to dispose effectively of inevitable medicine waste. This concerns the practices of drug logistics, drug household management, and medication management. These practices involve a large number of heterogeneous actors such as pharmaceutical manufacturers, wholesalers, retailers, logistics providers, general practitioners, community pharmacies, government, and consumers. These actors, together with their relations and interactions, constitute a complex socio-technical system.

Recently, IS researchers have described such dynamic complex systems as information infrastructures (IIs) by defining IIs as shared, open (and unbounded), heterogeneous and evolving sociotechnical systems [12]. However, there is very little research on how to connect practices which, so far, are entirely unrelated to one another. The literature focuses on cases where an information infrastructure grows gradually through extension 'at the edges' driven by user communities and enabled by modular and flexible designs (ibid.). However, information infrastructures may also develop in a more discontinuous, 'punctuated' manner [13]. This suggests that an information infrastructure may also emerge when three or more practices become related to one another which, so far, were unrelated. Drawing on a physics metaphor, the bringing together of several, so far unrelated practices may be sufficient to create a 'boiling point' where matter changes from one aggregate state to another. In this paper we therefore explore the facilitators and inhibitors of bringing together the three practices of waste drug recycling, medication management, and household drug management in order to understand the conditions under which the development of an information infrastructure, understood as an emergent phenomenon triggered by a re-configuration of practices, can happen.
We begin by presenting the literature on waste drug recycling, medication management and household drug management and also briefly summarize the literature on information infrastructure development. Next, we describe and analyze the current situation of drug waste management in China and outline the barriers to extending and broadening these practices and systems. The fourth section explores the possibilities and problems of connecting the three practices concerned: waste drug recycling, medication management, and household drugs management. We argue that by connecting the three practices the preconditions for the emergence of an innovative information infrastructure that supports expired drugs recycling can be created.

\section{Literature review}

\subsection{Waste drug recycling and drug review/ management systems}

To protect the environment, reduce economic loss and increase the safety of drug use, several international organizations have produced guidance information on managing waste medicines and on drug and medication review procedures [14, 15]. Some researchers advocate that an effective and welldesigned household waste drug recycling system can reduce avoidable waste medicines and effectively disposes of inevitable waste [1]. Kongar, Haznedaroglu [16] proposed a radio frequency identification-based information technology infrastructure for reverse logistics of end-of-life drugs products.

Although industries have various levels of involvement with waste drug recycling applications, such practices are regarded as difficult as they complicate the traditional forward-oriented medicine supply chain [17]. Recycling of waste medicines is challenging and difficult to implement because no commercial value can be recaptured from waste medicines. This causes hesitation and reluctance of the actors in the medicine supply chain to implement recycling processes [18]. This is quite a significant finding because it, in turn, inhibits initiatives to invest in the recycling infrastructure and related information systems and limits the scope for collaboration among actors. However, recycling packing materials, reuse of good condition medicines, sorting and separating processes of returned medicines etc. have become feasible practices to create residual value.

As a result of strict regulations and health and safety concerns, effective use and management of drugs have also become important for reducing avoidable waste, such as intervention and guidance in drug use, standardization of medicine package sizes, 
improving prescribing patterns, and storage management within household. With regard to waste drug management practices, the NHS (UK) in 2012 launched an action plan to determine how best practices can be shared to improve the use of medicines and to address medicine waste within the NHS [15]. The report also shows how improvements in the use of drugs have become an important part in the recycling of waste drugs.

With regard to research on medication reviews and management, in 2016 the Pharmaceutical Care Network Europe (PCNE) defined medication review as a structured evaluation of a patient's medicines with the aim of optimizing medicine use and improving health outcomes [14]. This entails detecting drug related problems and recommending interventions with regard to treatment effectiveness and avoidance of adverse drug events.

The main objective of drug management is to reduce financial expenditure, avoid waste, increase access, and ensure that drugs are properly used [19]. The so-called drug management cycle includes selection, procurement, storage and distribution, and use [20]. In the household context, drug management includes consumers' selection, purchase, storage, use, and disposal of drugs. Among these, the last item is related to drug recycling and the others are related to the medication review.

\subsection{Information infrastructure development}

The literature on information infrastructure design and development is largely in agreement with Hanseth and Lyytinen [12] characterization of information infrastructure as an open, shared, heterogeneous, and evolving socio-technical system. On this view, the development of an information infrastructure is not merely a switch between an old and a new information infrastructure, but a gradual and step-wise transition. Information infrastructure evolution is seen as an incremental, emergent process from simpler to more complex forms. Various authors have emphasized different aspects, such as the importance of standards [21], of the installed base [22], of modularity [23], of the role of users as designers [24], the flexibility of information infrastructure $[25,26]$, the role of governance structures [27], and the role of materiality [28]. However, the early emphasis on practices, as is evident in the foundational paper by Star and Ruhleder [29] has been largely lost. A view of information infrastructure as a constellation of practices has been proposed in the literature [30, 31] but not yet been widely adopted.

\section{The current situation of household waste drugs recycling in China}

In China, the recycling of expired drugs is still in an early stage. Some enterprises and governments at the local level are actively exploring and practicing different drug recycling models. These recycling models can be divided into two categories depending on who is the responsible actor, a pharmaceutical manufacturer (see Figure 1) or government (see Figure 2).

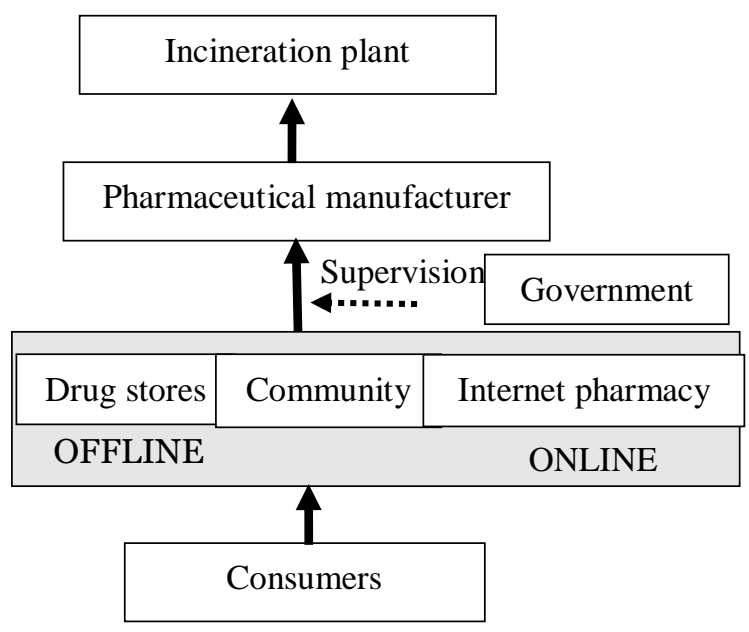

Figure 1. Pharmaceutical enterprise-led recycling for household waste drugs

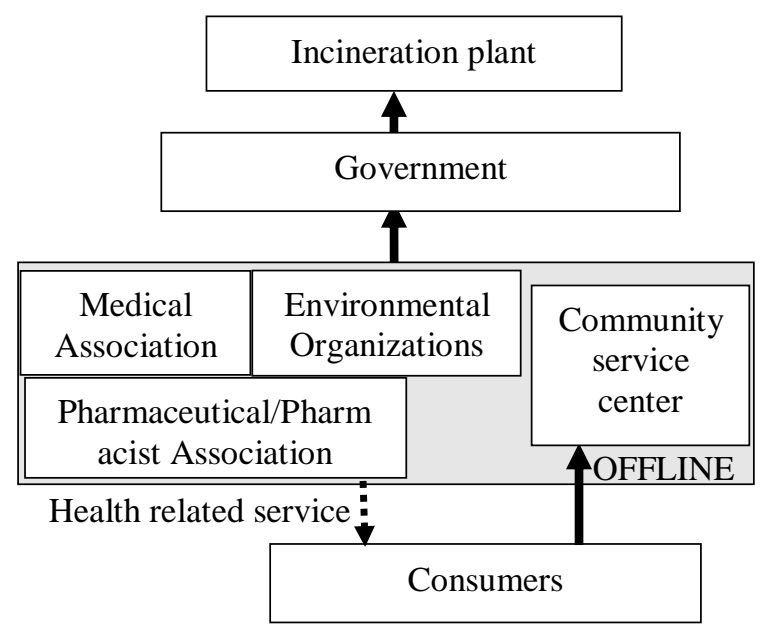

Figure 2. Government-led recycling for
household waste drugs

A typical example of the pharmaceutical enterpriseled recycling model concerns the system set up by Guangzhou Baiyunshan Pharmaceutical Holdings $(\mathrm{GBPH})$. Since the launch of an activity called "family 
expired drugs collection" in 2003 GBPH has carried out the activity regularly every year. Over the last 15 years, the company's recycling activities have been continuously extended. These extensions concern:

- the range of participating enterprises (from initially a single company to the whole group),

- the scope of operations (from a single locality to more than 200 cities nationwide),

- the number of participating drugstores (from initially five to more than 6000 pharmacies and community medical institutions),

- the reach of the service (from the doctor's waiting room to door-to-door visits for lonely elders in designated communities),

- the areas covered (from urban to rural),

- and the mode of recycling (from offline to online).

With the increasing awareness of the risks posed by expired drugs, more and more local governments have launched household expired drugs recycling activities and have made remarkable achievements in the improvement of recycling systems and the construction of expired drug collection points. Local governments have used various community centers as collection points for expired drugs. These collection points not only collect waste medicines but also provide some health-related service.

Despite such constant efforts there are still significant limitations concerning the existing models for recycling expired drugs. Table 1 shows the problems identified.

Table1. Problems identified

\begin{tabular}{|c|c|}
\hline Stakeholder & Problems identified \\
\hline $\begin{array}{l}\text { Waste drug recycling } \\
\text { enterprises }\end{array}$ & $\begin{array}{l}\text { Residual value is lacking } \\
\text { Recycling costs are high }\end{array}$ \\
\hline $\begin{array}{l}\text { Pharmacists and } \\
\text { doctors }\end{array}$ & Enthusiasm is low \\
\hline Consumers & $\begin{array}{l}\text { Collection points are } \\
\text { limited } \\
\text { Awareness is low } \\
\text { Enthusiasm is low } \\
\text { Trust is low }\end{array}$ \\
\hline Governments & Supervision is difficult \\
\hline
\end{tabular}

Firstly, there is no commercial value left in the current waste drug recycling system and the costs of recycling and disposal are very high; for example, GBPH invests tens of millions for its recycling activity every year [32]. Most of waste drugs have been collected in traditional ways and directly incinerated. This kind of model cannot recapture any value from returned drugs. Thus, there is only a very small number of large pharmaceutical companies carrying out recycling for a limited duration and with a limited range.

Secondly, there is no motivation for pharmacists and doctors to get involved in recycling activities. In government-led models, although the government is trying to ask pharmacists and doctors to provide health-related services in some community centers, there is no effective compensation mechanism. The activities are only known as charity.

Thirdly, consumers' awareness of possibilities to recycle drugs is low, together with the lack of incentives and of information sharing, which reduces consumers' willingness to contribute to drug recycling and their trust in recycling enterprises.

Finally, the lack of a tracking system makes it difficult for government to supervise drug recycling, leading to increases in the number of counterfeit drugs and creating an opening for a "grey" market for expired drugs to emerge. This has a negative impact on the environment, the financial situation of manufacturers, and causes social and health problems.

However, it seems that the current models offer some levers to improve this situation. In the manufacturer-led model, GBPH has begun to adopt an online model for recycling and is building consumers' electronic health profiles for some special groups (e.g., the elderly) since 2015. With the development of drug QR codes in China, possibilities for electronic management of expired drugs recycling and drug management are shaping up. Government is also trying to provide more health-related services in its own model. To improve consumers' awareness for the environment and their health, various specialists (e.g., pharmacists, environmental experts) provide drug and environment-related knowledge and advice. Thus, medication management is showing its role in the waste drug recycling process.

However, these practices have so far failed to evolve into an effective system for expired drugs recycling because they are not yet connected to each other. Following the clues provided by our analysis so far, we formulate:

Proposition 1. Effective practices for drug recycling require multi-actor involvement and crosspractice collaboration among the involved actors.

Considering the dynamic complexity involved in such an undertaking, we portray an evolutionary process from a single to a triple-practice system which could lead to the emergence of an information infrastructure that connects the practices of waste drug recycling, drug management, and medication management. 


\section{Emergence of an information infrastructure resulting from connecting the three practices}

\subsection{Information inf rastructure as a constell- ation of aligned practices}

As described above (see Section 2.2), information infrastructures are commonly defined as evolving complex systems consisting of heterogeneous technical and social components and as being characterized by an in principle unbounded functional scope and societal reach. However, information infrastructures continue to be understood as essentially socio-technical systems with some technical components at their core. By contrast, Star and Ruhleder [29] have argued for a practice-based understanding of information infrastructure, a proposal which has not yet been widely appropriated in the literature. For our purposes, however, a practice-based approach offers unique advantages as it allows for an understanding of an information infrastructure as a set or 'constellation' of aligned practices $[30,31]$. On such a view, an information infrastructure emerges or evolves through many small-scale changes in related practices so that relevant information can start to flow more easily across these practices. For example, in most practices the use of computers for information storage and processing is quite common. However, sharing such information across practices often encounters formal and informal barriers. A traditional information systems approach would call for the definition of clear interface specifications so that information can flow more efficiently between related systems. However, a practice-based approach opens up many more possibilities, not all of which are technical in nature. Oftentimes, miniscule adaptations in system settings, coding procedures or data maintenance operations may be all that is needed in order to improve the flow of information across practices. While such changes may appear as insignificant and trivial when taken individually, in sum they may have far reaching effects. Discovering such opportunities for adaptation, however, is not an easy task. Members of one practice may have to improve their awareness and understanding of how related practices operate and 'function'. Thus, a better mutual understanding of related practices may be the most important first step towards making the many small-scale changes that, together, lead to the evolution or emergence of an information infrastructure, understood as a constellation of aligned practices. In the following we are guided by this understanding of information infrastructure which takes as its point of departure not a technical system that needs to be connected with other technical systems but a practice that needs to be better aligned with related practices.

\subsection{Methodological approach}

Due to the growing need for coordination and collaboration among various actors, the process of information infrastructure evolution and emergence is characterized by a high degree of unpredictability. Gray and Hovav [33] have introduced the use of scenarios to the IS field to address such uncertainty. Specifically, they argue that scenarios provide a legitimate and practical "way of communicating about the future" (1999, p. 15). Scenarios are not to be confused with predictions; rather, they help managers and politicians to understand the assumptions and implications of various possible courses of actions and thus to prepare for the future without having to predict it (1999, p. 15).

Identifying key drivers is crucial for creating a scenario space [33]. In the following, we will apply this insight to develop a plausible scenario for evolving an information infrastructure for recycling expired drugs based on the assumption that such evolution is driven by the possibilities that result from connecting the involved practices in multiple ways. We will develop this scenario in three steps by gradually extending the number of connections between the three practices.

\subsection{Single practice scenario}

The first scenario views the three practices of waste drug recycling, household drug management, and medication management as independent and unconnected, as shown in Figure 3. This represents the current situation.

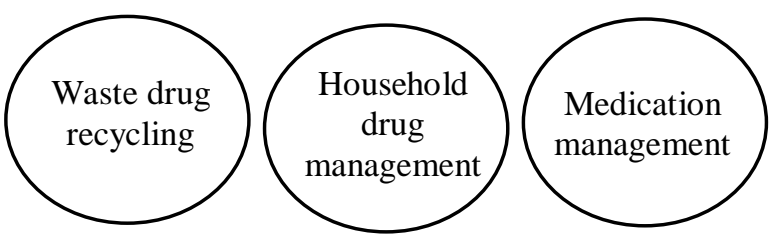

Figure 3. Three independent practices

4.3.1. Waste drug recycling. Waste drug recycling involves manufacturers, wholesalers, retailers, logistics providers, and consumers. The objective of the system is to recycle the expired drugs from consumers. Based on some experiences in some developed countries, returned drugs are special types of end-of life (EoL) or end-of-use (EoU) returns of consumer goods [34]. 
Some waste medicines, as EoU returns, still preserve functionality intact and are capable of being reused [35]. Package-related returns bring some residual value and create useful information which can influence the package sizes, shapes, materials used and so on. Drug recycling enterprises optimize the package design to protect products properly and allow consumers to use the product safely.

Electronic order management is beneficial for logistics companies to optimize transportation, for manufacturers and wholesalers to obtain expired drugrelated data to guide pharmaceutical research, development, design, and sales.

Retailers, as collection points, interact directly with consumers. By integrating consumers' purchase records with recycling records, they can offer personalized recommendations and improve quality of service. In some cases, retailers provide promotional activities, such as discount coupons, bonuses, etc. to improve consumers' awareness of possibilities to return expired drugs.

However, these measures provide only limited value for participants. Due to strict regulations and health and safety concerns, the impact of actual cost savings per returned product for waste drugs recycling management is small [8]. Sorting and separating processes for returned medicines can be complex, and often only a minority of products are intact, in good condition and in shelf life [8]. This means that the extent to which reuse of medicines can affordably reduce the costs of medicine waste is likely to be very small. Thus, it is crucial to find more complementary measures to improve the motivation of waste drugs recycling enterprises. More effective savings would be generated from improved use of drugs [15], greater standardization of medicine package sizes, and improved prescribing patterns [1], all of which would reduce avoidable waste in a cost-effective manner.

4.3.2. Household drug management. Household drug management is related to consumers. Consumers can manage their own drugs, such as inputting drug information into a computerized inventory management system and obtaining basic instructions from drug booklets about classification, precautions and so on. However, due to the special nature of drugs, especially health and environmental risks associated with drug use, it is important that professional actors (e.g. pharmacists or doctors) provide effective drug use instruction and help.

4.3.3. Medication management. Pharmacists and doctors adopt medication management systems to evaluate and intervene in patients' medication with regard to treatment effectiveness and avoidance of adverse drug events. Thus, drug use-related information is an important resource for these two actors' medication management. However, in the independent system context, this information is rare because it comes from external sources, such as manufacturers and consumers.

\subsection{Two-practice scenario}

The second scenario shows the interaction between any pair of practices through sharing information and providing service to create incentives for participants in these practices, as shown in Figure 4.

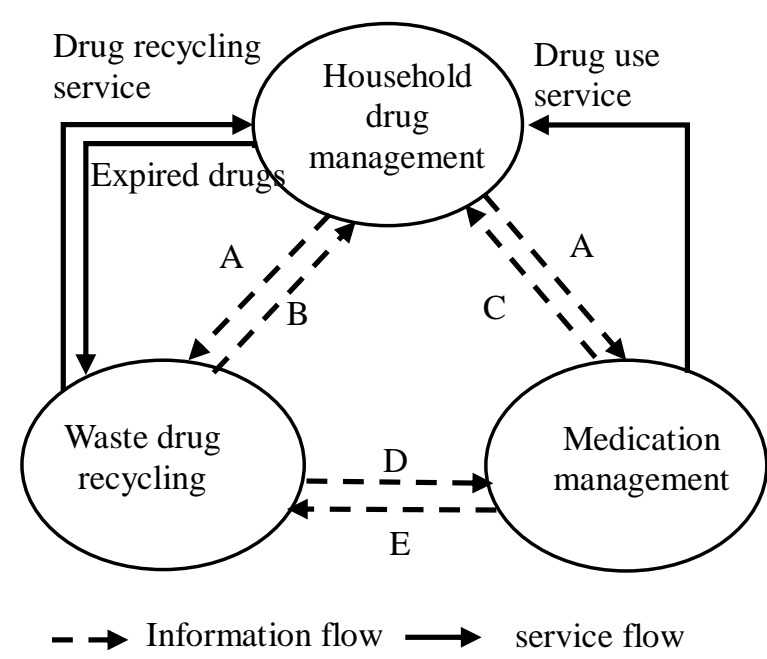

A: consumer's unexpired/expired/unwanted drug information

B: recycling information, e.g., collection points, how to dispose of expired drugs

C: drug use information, e.g. how to use, store, and dispose of drugs

D: expired drugs

E: practical efficacy of drugs

\section{Figure 4. The interaction between pairs of two practices}

4.4.1. Waste drug recycling and household drug management. In scenario 1, waste drug recycling only focuses on collection and disposal of expired drugs. The value of such a system is likely to be small. Research findings suggest that waste drugs recycling cannot happen unless the consumer is compliant and becomes involved, e.g. to return the expired/unwanted drugs to the collection points [36]. In waste drugs recycling, the role of the consumer is not only as a consumer of drug/medication services, but also as an actor to reduce unneeded drugs and to return the unwanted drugs. Consumers' expectations towards receivers accepting these returns form an important 
noneconomic aspect of consumer service [37]. Consumers need to be educated to manage unwanted medication differently, e.g. to bring the drugs to a collection site or hazardous waste facility rather than dispose of medication into water or domestic waste systems.

In scenario 2, through connecting household drugs management and waste drug collection practices, waste drugs recycling can provide recycling related information, e.g. at the collection point, reminding patients of a drug's expiration date and proper ways of disposal, which can have a significant influence on increasing product returns from consumers and reduce recycling costs and the waste itself. Further, waste drugs recycling enterprises can obtain information about unexpired/unwanted drugs from consumers that further guide drug research, development, design and sales.

Consumers can be offered more effective recycling waste drugs service. The drugs recycling enterprises provide offline and online collection channels. Consumers can find the nearest collection point to send waste drugs to or order expired drugs pick-up in their homes online. Consumers can track the process of drug disposal and share drug-related information, which improves consumers' trust and involvement.

Proposition 2. The connection between waste drugs recycling and household drug management practices has a significant positive impact on reducing recycling costs and waste and promotes consumers' involvement and trust.

4.4.2. Waste drug recycling and Medication management. In scenario 1 , for independent medication management it is difficult to obtain drugrelated information because the information comes from external sources, such as manufacturers and consumers. Manufacturers have the initial instruction regarding drug usage and consumers have experiences with drugs. Waste drug management produces much valuable information related to expired drugs. The information is also an important resource for medication management. For example, based on the causes of expired drugs, doctors can improve prescribing patterns and pharmacists can provide effective advice to reduce waste.

In addition, the independent waste drug recycling enterprises, e.g. manufacturers, are particularly concerned about the practical efficacy of drugs because this is directly related to their pharmaceutical research, development and sales.

Proposition 3. The connection between waste drugs management and medication management has a significant positive impact on improving the efficacy and effective use of drugs.
4.4.3. Household drug management and Medication management. As an indispensable resource, consumers' drug and drug use-related information has a significant influence on medication management. Household drugs management provides unexpired and expired drug information which helps pharmacists make more effective evaluation and give better medication advise. In turn, effective drug-related guidance, e.g. how to use, store, and dispose of the drugs, raises consumers' awareness of the impact of drugs on the environment and their health, which increases the willingness of patients to return drugs and to reduce waste. There is a need to increase consumers' loyalty for the success and sustainability of medication management.

Proposition 4. The connection between household drug management and medication management has a significant positive impact on improving the effective use of drugs and consumers' awareness of environmental and health risks associated with drug use.

\subsection{Three-practice scenario}

In scenario 3, a platform emerges which simplifies information sharing among the three practices, strengthens and enriches interactions among them, and draws in further actors (e.g., government and environmental protection organizations), as shown in Figure 5.

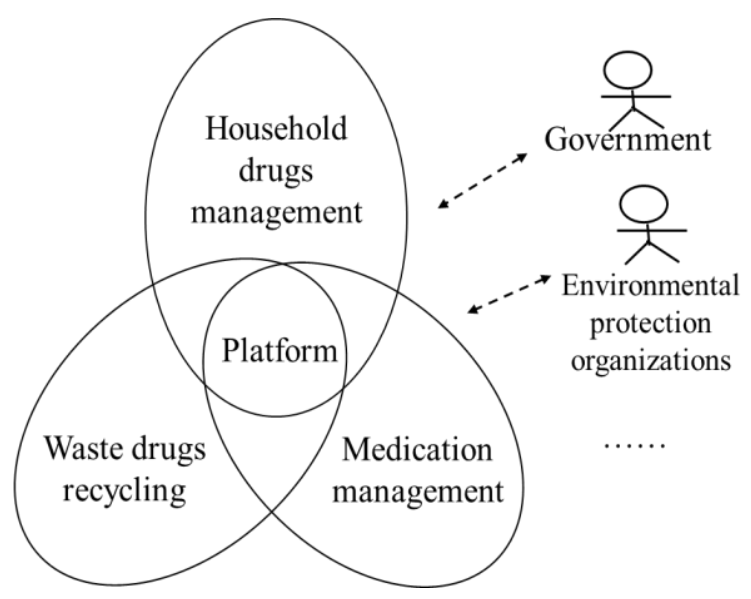

Figure 5. The three-practice scenario

In scenario 2, although the interaction between any pair of two practices is beneficial to each participant, the redundancy of information and the complexity of communication act as barriers for the creation of further connections between practices. For all information, each role needs to share and communicate 
with others separately; for example, consumers' drug information is transferred to waste drug recycling and medication management separately. By contrast, the three-practice scenario opens up a space for many more connections as a platform emerges which also has the potential to pull in further actors such as government and environmental protection organizations.

All of the participants need more and better drugrelated information to realize their own objectives. For "creating" residual value, reducing waste and cost, waste drugs recycling enterprises need to obtain drugrelated information from both household drugs and medication management. Correspondingly, for improving effective use of drugs there is a need to obtain more drug use-related information from manufacturers and consumers. Consumers' unexpired and expired drugs need to be managed effectively by both recycling enterprises and pharmacists. Thus, it becomes important to create additional connective ways between the three practices to strengthen and enrich their communication and collaboration. A platform will emerge from pairs of connections to integrate redundant information and enrich interactions among all actors. For example, information on how to effectively use a drug or a class of drugs, how to dispose of drugs, and on drug quality certification could be shared among all participants. At the same time, information security and privacy protection caused by information sharing can be addressed more effectively.

In addition, the three-practice scenario not only expands existing services and the user base but also increases its scope and reach by including new actors. For example, government can now realize a more effective supervision to strengthen drug safety, information security, and privacy protection because of better integration of unexpired/expired drugs information and medication management. Moreover, in recent years environmental protection organizations have become more concerned about expired drugs and their possible negative impact on soil and water because pharmaceutical contamination is becoming ubiquitous in the environment. As pharmaceutical contamination cannot be effectively removed from wastewater by conventional methods, the occurrence of expired drugs has received increasing attention by environmental protection organization [38]

Proposition 5. As the number of connections between practices increases, a platform emerges that simplifies and enriches the connections between all three practices and thus creates even more possibilities for expanding existing services and the user base, including opportunities to increase the scope of the network and the range of stakeholders.

\section{Conclusions}

Our analysis and scenarios have shown that there is a significant potential to increase the motivation for all actors concerned with expired drugs recycling by connecting the three involved practices of waste drug recycling, medication management, and household drug management in manifold ways. From a practical perspective, connecting the three practices in this manner would greatly contribute to effectively addressing a growing problem and burden for the environment resulting from increasing usage of medicines by an aging and more affluent population. From a theoretical perspective, our analysis suggests a novel mechanism for information infrastructure emergence. While information infrastructure development has so far been portrayed as primarily driven through growth at the periphery, mostly through user activity enabled by modular and flexible designs, an information infrastructure may also emerge de novo as a result of connecting so far unrelated practices in a sufficiently rich and multi-directional manner, similar to the phenomenon of state transitions known from physics. Our scenarios show how such a 'phase transition', i.e. the phenomenon of emergence, might come about for the case of information infrastructures.

\section{Acknowledgments}

We gratefully acknowledge support by the German Federal Ministry for Research and Education (BMBF) (Grant 01DO17024A/B) and the National Natural Science Foundation of China (Grant 71402159).

\section{References}

[1] Y. Xie and B. Liz, "Who cares wins? A comparative analysis of household waste medicines and batteries reverse logistics systems: The case of the NHS (UK)", Supply Chain Management: An International Journal, 19(4), 2014, pp. 455474.

[2] C.G. Daughton and T.A. Ternes, "Pharmaceuticals and personal care products in the environment: agents of subtle change?", Environmental Health Perspectives, 107(6), 1999, pp. 907-938.

[3] T.A. Ternes, "Occurrence of drugs in German sewage treatment plants and rivers", Water Research, 32(11), 1998, pp. 3245-3260.

[4] T.A. Ternes, et al., "Behavior and occurrence of estrogens in municipal sewage treatment plants-I. Investigations in Germany, Canada and Brazil", Science of the Total Environment 225(1-2), 1999, pp. 91-99.

[5] H.R. Buser, M.D. Müller, and N. Theobald, "Occurrence of the Pharmaceutical Drug Clofibric Acid and the Herbicide Mecoprop in Various Swiss Lakes and in the North Sea", 
Environmental Science \& Technology, 32(1), 1998, pp. 188192.

[6] T. Heberer, "Occurrence, fate, and removal of pharmaceutical residues in the aquatic environment: a review of recent research data", Toxicology Letters, 131(1), 2002, pp. 5-17.

[7] Guangzhou Phamaceutical Holdings (GPH), http://www.gpc.com.cn/article/12134.html.

[8] Department of Health (DoH), "Evaluation of the Scale, Causes and Costs of Waste Medicines", www.gov.uk/ government/publications/making-best-use-ofmedicinesreport-of-a-department-of-health-roundtableevent-hosted-bythe-king-s-fund, accessed 30 November 2013.

[9] C.J. Savage, K.J. Roberts, and X.Z. Wang, "A Holistic Analysis of Pharmaceutical Manufacturing and Distribution: Are Conventional Supply Chain Techniques Appropriate?", Pharmaceutical Engineering, 26(4), 2006, pp. 10-18.

[10] J.M. Pomerantz, "Recycling expensive medication: Why not?", Medscape General Medicine, 6(2), 2004, pp. 4.

[11] K. Kümmerer, "Pharmaceuticals in the Environment", Regulatory Toxicology \& Pharmacology, 28(3), 2015, pp. 212-221.

[12] O. Hanseth and K. Lyytinen, "Design theory for dynamic complexity in information infrastructures: the case of building internet", Journal of Information Technology, 25(1), 2010, pp. 1-19.

[13] K. Lyytinen and M. Newman, "Explaining information systems change: a punctuated socio-technical change model", European Journal of Information Systems, 17(6), 2008, pp. 589-613.

[14] Pharmaceutical Care Network Europe (PCNE), http://www.pcne.org/upload/files/149_Position_Paper_on_P CNE_Medication_Review_final.pdf, 2016

[15] Department of Health (DoH), "Improving the use of medicines for better outcomes and reduced waste: an action plan", https://assets.publishing.service.gov.uk/government/ uploads/system/uploads/attachment_data/file/212837/

Improving-the-use-of-medicines-for-better-outcomes-andreduced-waste-An-action-plan.pdf.

[16] E. Kongar, et al., "A novel IT infrastructure for reverse logistics operations of end-of-life pharmaceutical products", Information Technology and Management, 16(1), 2015, pp. 51-65.

[17] X. Li and F. Olorunniwo, "An exploration of reverse logistics practices in three companies", Supply Chain Management, 13(5), 2008, pp. 381-386.

[18] C. Prahinski and C. Kocabasoglu, "Empirical research opportunities in reverse supply chains", Omega, 34(6), 2006, pp. 519-532.

[19] WHO, "Management of Drugs at Health Centre Level", http://apps.who.int/medicinedocs/pdf/s7919e/s7919e.pdf.

[20] MSH/WHO, Managing Drug Supply, Kumarian,West Hartford, Connecticut, 1997.

[21] O. Hanseth, E. Monteiro, and M. Hatling, "Developing Information Infrastructure: The Tension Between Standardization and Flexibility", Science, Technology, \& Human Values, 21(4), 1996, pp. 407-426.

[22] O. Hanseth, Developing Pan-European e-Government Solutions: From Interoperability to Installed Base Cultivation, in The Circulation of Agency in E-Justice. Law, Governance and Technology Series, C. F. and L. G., Editors. 2014, Springer: Dordrecht

[23] M. Aanestad and T.B. Jensen, "Building nation-wide information infrastructures in healthcare through modular implementation strategies", The Journal of Strategic Information Systems, 20(2), 2011, pp. 161-176.

[24] O. Hanseth and N. Lundberg, "Designing Work Oriented Infrastructures", Computer Supported Cooperative Work (CSCW), 10(3), 2001, pp. 347-372.

[25] M. Grisot, O. Hanseth, and A.A. Thorseng, "Innovation of, in, on infrastructures: Articulating the role of architecture in information infrastructure evolution", Journal of the Association for Information Systems, 15(4), 2014, pp. 197219.

[26] J. Braa, et al., "Developing Health Information Systems in Developing Countries: The Flexible Standards Strategy", Mis Quarterly, 31(2), 2007, pp. 381-402.

[27] P. Constantinides and M. Barrett, "Information Infrastructure Development and Governance as Collective Action", Information Systems Research, 26(1), 2015, pp. 4056.

[28] W. Venters, E. Oborn, and M. Barrett, "A trichordal temporal approach to digital coordination: the sociomaterial mangling of the CERN grid", MIS Quarterly, 38(3), 2014, pp. 927-949.

[29] S.L. Star and K. Ruhleder, "Steps Toward an Ecology of Infrastructure: Design and Access for Large Information Spaces", Information Systems Research, 7(1), 1996, pp. 111134.

[30] R. Kai, R.B. Johnston, and S. Klein, "The difficulty of studying inter-organisational IS phenomena on large scales: critical reflections on a research journey", Electronic Markets, 20(3-4), 2010, pp. 229-240.

[31] R. Kai, R.B. Johnston, and S. Klein, "An empirical evaluation of existing IS change theories for the case of IOIS evolution", European Journal of Information Systems, 23(4), 2014, pp. 373-399.

[32] Guangzhou Phamaceutical Holdings (GPH), http://www. gzgz.gov.cn/gzsgzw/shzrzdgz/201803/7bf025eee5484bc2979 ded41bfeedbdf.shtml, 2018

[33] P. Gray and A. Hovav, "Using scenarios to understand the frontiers of IS", Information Systems Frontiers, 1(1), 1999, pp. 15-24.

[34] E.J. Marien, "Reverse logistics as competitive strategy", Supply Chain Management Review, 34(2), 1998, pp. 43-52.

[35] H. Krikke, I.l. Blanc, and S.v.d. Velde, "Product Modularity and the Design of Closed-Loop Supply Chains", California Management Review, 46(2), 2004, pp. 23-39.

[36] Y. Xie and B. Liz, "Greening community pharmaceutical supply chain in UK: a cross boundary approach", Supply Chain Management: An International Journal, 17(1), 2012, pp. 40-53.

[37] S. Dowlatshahi, "Developing a Theory of Reverse Logistics", Interfaces, 30(3), 2000, pp. 143-155.

[38] J. Wang and S. Wang, "Removal of pharmaceuticals and personal care products (PPCPs) from wastewater: A review", Journal of Environmental Management, 182(2016, pp. 620640. 